\title{
A model-driven approach for enforcing summarizability in multidimensional modeling
}

\author{
Jose-Norberto Mazón ${ }^{1}$, Jens Lechtenbörger ${ }^{2}$, and Juan Trujillo ${ }^{1}$ \\ 1 Lucentia, Dept. of Software and Computing Systems, University of Alicante, Spain \\ \{jnmazon, jtrujillo\}@dlsi.ua.es \\ 2 Dept. of Information Systems, University of Münster, Germany \\ lechten@wi.uni-muenster.de
}

\begin{abstract}
The development of a data warehouse system is based on a conceptual multidimensional model, which provides a high level of abstraction in the accurate and expressive description of real-world situations. Once this model has been designed, the corresponding logical representation must be obtained as the basis of the implementation of the data warehouse according to one specific technology. However, there is a semantic gap between the dimension hierarchies modeled in a conceptual multidimensional model and its implementation. This gap particularly complicates a suitable treatment of summarizability issues, which may in turn lead to erroneous results from business intelligence tools. Therefore, it is crucial not only to capture adequate dimension hierarchies in the conceptual multidimensional model of the data warehouse, but also to correctly transform these multidimensional structures in a summarizability-compliant representation. A model-driven normalization process is therefore defined in this paper to address this summarizability-aware transformation of the dimension hierarchies in rich conceptual models.
\end{abstract}

\section{Introduction}

Business intelligence tools, such as OLAP (On-Line Analytical Processing) tools depend on the multidimensional (MD) structures of a data warehouse that allow analysts to explore, navigate, and aggregate information at different levels of detail to support the decision making process. Current approaches for data warehouse design advocate to start the development by defining a conceptual model in order to describe real-world situations by using MD structures [13]. These structures contain two main elements. On one hand, dimensions which specify different ways the data can be viewed, aggregated, and sorted (e.g., according to time, store, customer, product, etc.). On the other hand, events of interest for an analyst (e.g., sales of products, treatments of patients, duration of processes, etc.) are represented as facts which are described in terms of a set of measures. Every fact is based on a set of dimensions that determine the granularity adopted for representing the fact's measures. Dimensions, in turn, are organized as hierarchies of levels that allow analysts to aggregate data at 
different levels of detail. Importantly, dimension hierarchies must ensure summarizability, which refers to the possibility of accurately computing aggregate values with a coarser level of detail from values with a finer level of detail. If summarizability is violated, then incorrect results can be derived in business intelligence tools, and therefore erroneous analysis decisions [3,4]. In addition, summarizability is a necessary precondition for performance optimizations based on pre-aggregation [10].

Usually, summarizability are not solved at the conceptual level, but at late stages of the development by using information from the data contained in the implemented data warehouse $[11,9]$. This way of proceeding poses problems to data warehouse designers, since great efforts are required to ensure summarizability due to the huge amount of data stored. However, if one tries to ensure summarizability at the conceptual level, one typically obtains MD models that are more difficult to understand as they contain an excessive amount of detail that is not required in the initial design steps. As understandability must be one inherent property of conceptual MD models, designers should be able to specify rich conceptual models without being concerned with summarizability problems. Then, a normalization process should be applied to transform the designed MD model into a constrained conceptual model, which is restricted to those MD structures that do not violate summarizability. This normalized model should also provide a high level of expressiveness in describing real-world situations.

Bearing these considerations in mind, in this paper, we take advantage of model-driven development to propose a comprehensive normalization process by using widely adopted formalisms. In essence, we describe how to (i) design different kinds of dimension hierarchies in a conceptual model in order to easily and understandably represent real-world situations regardless of summarizability problems, and (ii) automatically derive equivalent normalized conceptual models, which are constrained to those kind of dimension hierarchies that do not violate summarizability. From this normalized MD model, an implementation that satisfies summarizability can be easily deployed in any database platform and can be accurately queried by any business intelligence tool.

The rest of this paper is structured as follows. Related work is described in Section 2. Our normalization process for ensuring summarizability in dimension hierarchies is presented in Section 3. Finally, conclusions and potential for future work are provided in Section 4.

\section{Related Work}

In [9] we present a survey on summarizability issues in MD modeling. Briefly, MD modeling aims to represent measurable facts for real-world events under different perspectives of detail, which are specified via dimensions. For example, using the UML profile for MD modeling proposed in [5], Fig. 1 represents Sales via a Fact (馟) class and Date, Product, and Customer via Dimension ( composed of measures or fact attributes, which are represented as attributes with the FactAttribute stereotype (FA) such as Price and Quantity in Fig. 1. Moreover, 
dimension levels, which allow to analyze measures at a specific level of detail, are specified by Base classes ( $\boldsymbol{B}$ ) such as Day and Week. Associations (represented by the stereotype Rolls-UpTo, (C) between pairs of Base classes form dimension hierarchies. Importantly, Rolls-UpTo associations between Base classes as well as associations between Fact and Dimension classes are annotated with UML multiplicities. E.g., the multiplicities for the association between Country and Region in Fig. 1 indicate that every region belongs to exactly one country ("1" in role $r$ at the Country end) whereas there are countries (such as "Andorra", "Monaco", etc.) without associated regions ("0..*" in role $d$ at the Region end). Finally, UML generalization relationships between Base classes can be used to represent optional dimension levels within a hierarchy.

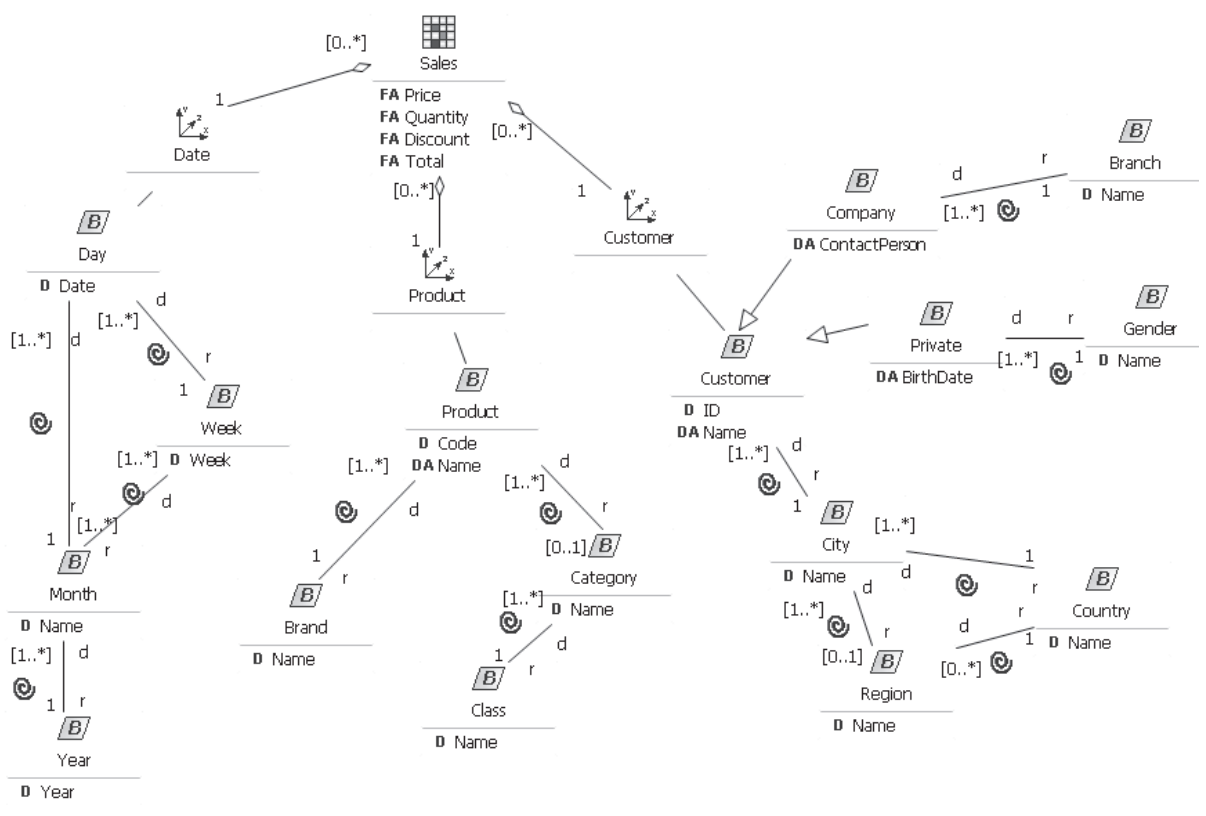

Fig. 1. Sample scenario.

In the context of MD data, summarizability presents a guarantee concerning the correctness of aggregated measures. The notion of summarizability was originally introduced in [12] and later characterized in [4]. As observed in [9], minimum and maximum multiplicities of associations characterize sub-structures where summarizability is violated. In earlier work [8], we have shown how to normalize MD schemata with non-summarizable fact-dimension associations into summarizable ones. With respect to non-summarizable Rolls-UpTo associations, however, a similar approach still needs to be designed. 
Indeed, in a fundamental work Pedersen et al. $[10,11]$ propose instance level algorithms to automatically transform dimension hierarchies to achieve summarizability. As this proposal works at the instance level, it is necessary to transform the data that will populate the DW, which may involve considerable efforts of preprocessing. In particular, ETL processes become more complex, as summarizability checks must be incorporated and executed for every update. In addition, as the data transformations produce artificial data values, data analysis becomes more complex.

In $[6,7]$ the authors present a classification of different kinds of complex dimension hierarchies, and they define the MultiDimER model for the conceptual design of complex MD models based on an extension of the well-known EntityRelationship (ER) model. The idea is that this classification guides developers to properly capture at a conceptual level the precise semantics of different kinds of hierarchies without being limited by current data analysis tools. Furthermore, the authors discuss how to map these conceptual hierarchies to the relational model (enabling implementation in commercial tools). Unfortunately, the mapping between the conceptual and the logical level is described informally. In addition, the commented mapping is tool-dependent and it may vary depending on the scenario.

\section{Model-driven development of dimension hierarchies}

While a conceptual MD model should represent the real world in a way that is easy to understand and that supports discussions with end users, a logical MD model must support the implementation in a chosen target technology in such a way that data can be accurately analyzed. To bridge this semantic gap, we propose a model-driven normalization process to derive an intermediate model, which should still be expressed at the conceptual level (to avoid limitations imposed by particular target models) but where MD structures that violate summarizability conditions are replaced with summarizable alternatives (which are typically more precise as we will see below). In particular, we address the three types of non-summarizable Rolls-UpTo associations enumerated in [9]: Non-strict, roll-up incomplete, drill-down incomplete

Non-strict associations are those where the maximum multiplicity at role $r$ is * (instead of 1). For example, the association between Week and Month in Fig. 1 is non-strict, and requires special care to avoid the well-known double counting problem. Next, an association is drill-down incomplete if the minimum multiplicity at role $d$ is 0 ; otherwise, it is drill-down complete. For example, the association between Country and Region in Fig. 1 is drill-down incomplete as there are countries (such as "Andorra", "Monaco", etc.) without associated regions. As explained in [9] in this case one has to be careful when drilling down from aggregate values at the level Country towards the level Region as values for countries without regions may not be accounted for, leading to inconsistent grand totals. Finally, an association is roll-up incomplete if the minimum multiplicity at role $r$ is 0. For example, the association between Product and Category in 
Fig. 1 is roll-up incomplete. As explained in [9] in this case one has to be careful when rolling up from aggregate values at the level Product towards the level Category as values for products that are not assigned to any category will not be accounted for, leading again to inconsistent grand totals.

In the following subsections, we show how to carry out a normalization process to obtain a conceptual MD model that ensures summarizability while accurately capturing the expressiveness of the demanded real-world situation. This process is composed of a set of transformations to obtain a normalized MD model constrained to hierarchies that contain only those elements and relationships that do not violate summarizability. For the sake of understanding, these transformations are first defined informally and only two of them are formally described in QVT (Query/View/Transformation) due to space constraints.

\subsection{Eliminating Non-Strictness}

Non-strictness is eliminated by one transformation which is based on one QVT relation (named as nonStrictBases) that replaces all occurrences of non-strict Rolls-UpTo associations in the source model with constructs that do not violate summarizability. The rationale behind this QVT relation is as follows. A nonstrict association, i.e., a many-to-many relationship among dimension instances, represents the least restrictive possible case. In fact, even if no association is declared among Base classes then adding a non-strict (and incomplete) association does not impose any additional restriction on the instances. E.g., in Fig. 1 every Product has a Brand and may have a Category. As the relationship among Brand and Category is not declared explicitly, we may reasonably assume to find many Brands associated with the Products for each Category and vice versa. Hence, we may safely add a non-strict association from Category to Brand without changing the meaning of the model.

More generally, consider a non-strict association between Base classes $b_{1}$ and $b_{2}$. On the one hand, this association is redundant and can be removed safely if there are alternative strict Rolls-UpTo associations to $b_{2}$ (which is the Base class that causes the non-strictness). On the other, the association cannot be removed if the Base class $b_{2}$ does not play role $r$ in some strict association. In this latter case, removing the association would isolate $b_{1}$ from $b_{2}$. Moreover, in this case the instances of $b_{2}$ are in a many-to-many relationship not only with $b_{1}$ but also with all Base classes that roll-up from (possibly transitively) $b_{1}$. This many-to-many relation is naturally expressed by moving $b_{2}$ into a newly created dimension, which again leads to the removal of the non-strict association.

As an example for a many-to-many relation that should be represented via a newly created dimension, assume that Products in the scenario of Fig. 1 are books for which the authors are of interest. Then, we may add the Base class Author along with a non-strict association from Book to Author (see Fig. 2(a)).

Although such an association allows to represent the relationship between books and their authors, this relationship is unrelated to the sales of individual books. Besides, computing sales volumes per author based on that relationship may easily lead to summarizability problems due to double counting. Hence, our 


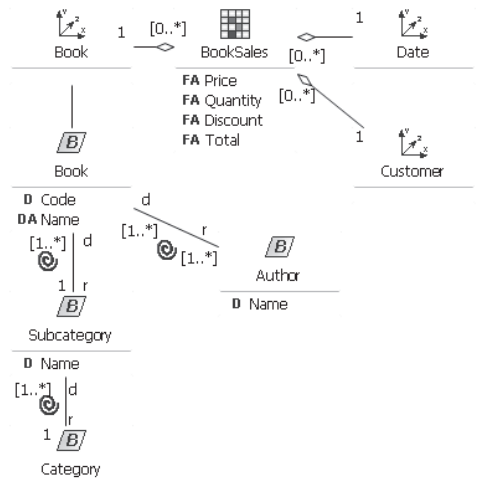

(a) Excerpt of the source model.

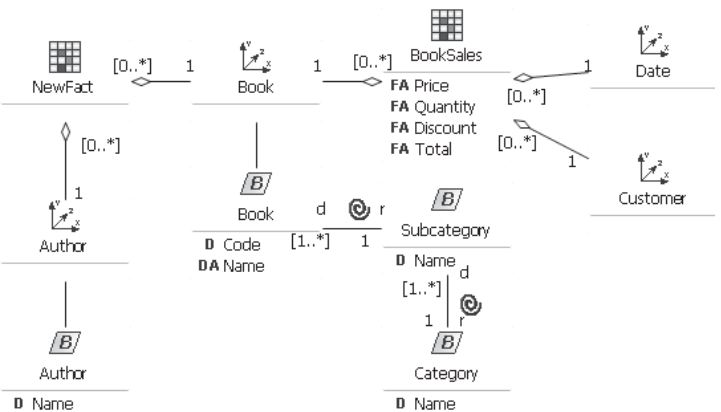

(b) Excerpt of the target model.

Fig. 2. Newly created dimension for eliminating non-strictness: the Book sales example.

normalization process removes Author from the Fact class Sales and transforms the non-strict association into the natural many-to-many relationship of a newly created fact, in this example into a two-dimensional Fact class NewFact with Dimension classes Book and Author. As Dimension class Book is common to both facts, queries involving Sales for Authors are still possible via drill-across operations. Moreover, to support summarizable queries, the designer may want to manually add additional measures, e.g., each author's share per book, to the new fact schema. The target model is shown in Fig. 2(b).

The nonStrictBases relation is shown in Fig. 3(a). It checks that there is a non-strict Rolls-UpTo association between two Base classes $\left(b_{1}\right.$ and $\left.b_{2}\right)$ in the source model (multiplicity $*$ in the role $r$ ) in order to create the appropriate elements in the target model to obtain a representation without non-strict associations. The two cases concerning the treatment of non-strict associations explained above are captured by a condition that must hold to execute this relation (see when clause of Fig. 3(a)). This condition checks whether Base class $b_{2}$ plays role $r$ in some strict association in the source model. On the one hand, if this condition does not hold then the QVT relation is not launched, and no new Rolls-UpTo association is created in the target model, since the two Base classes are related via a many-to-many relationship by default. On the other, if the condition is satisfied then $b_{2}$ does not play role $r$ in some strict association. In this case, a new Fact class $f$ is created which is associated with two new Dimension classes: $d_{1}$ and $d_{2}$. Specifically, $d_{1}$ corresponds to the Dimension class related to the Base classes $b_{1}$ and $b_{2}$ of the source model, while $d_{2}$ is a new Dimension class whose defining Base class is $b_{2} p$ (which is a copy of the Base class that causes the non-strictness in the source model $b_{2}$ ).

After the execution of the nonStrictBases relation, an excerpt of the target model related to the Date dimension is shown in Fig. 4(a). Here, the non-strict Rolls-UpTo association between Month and Week has disappeared, since in this 


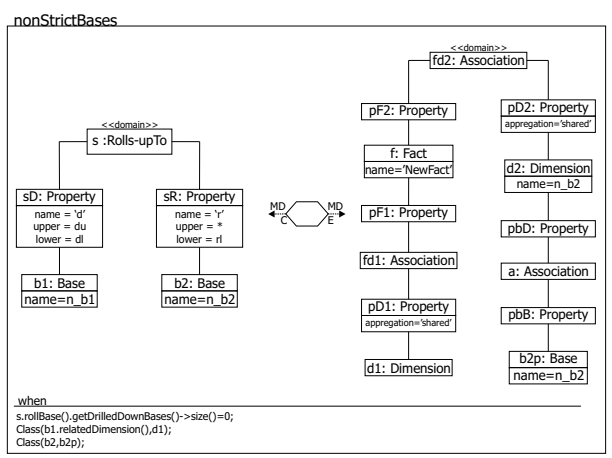

(a) Eliminating non-strictness.

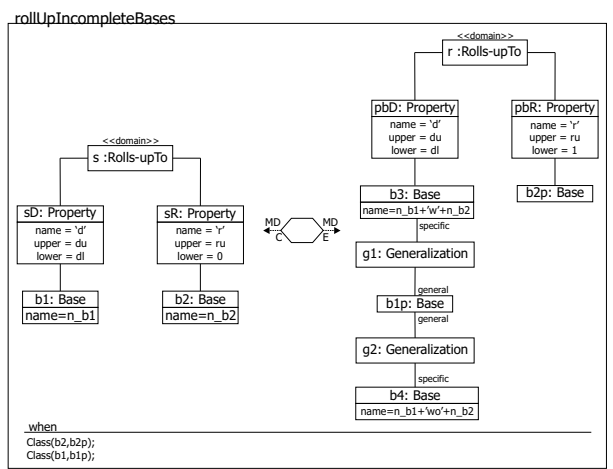

(b) Eliminating roll up incompleteness.

Fig. 3. QVT relations for normalizing hierarchies.

way both Bases classes are already many-to-many related, and there is no need for the creation of a new Fact class. The remaining parts of the source model that do not present non-strictness problems are copied directly to the target model. It is worth noting that all the possible strict Rolls-UpTo association between Base classes are assumed to be explicitly modeled in the source model and they will also appear in the target model. In this way, analysis capabilities of the source model are not negatively affected. Anyway, if users would still like to navigate between aggregate values by means of a non-strict relationship, then a new type of association could be added to the conceptual model; such "navigational" arcs, however, would need special support from implementing OLAP tools and are beyond the scope of this paper.

With respect to the soundness of the nonStrictBases relation shown in Fig. 3(a) we focus on the non-strict association of the source model, say association $s$ between $b_{1}$ and $b_{2}$, which is represented differently in the target model. We consider two cases: First, the QVT relation just removes the non-strict association. In this case, the when clause of the relation assures that $b_{2}$ is alternatively reachable via strict Rolls-UpTo associations. Hence, in the target model both Base classes still belong to a common dimension hierarchy, where all pairs of Base classes are related via many-to-many relationships by default as we have explained above, and there is no loss of information when removing the non-strict association. Second, the QVT relation creates a new Fact class to represent the many-tomany relationship. In this case, the associations between instances of $b_{1}$ and $b_{2}$ of the source model are simply stored as instances of the new Fact class in the target model. Hence, the information that can be represented under the source model can also be represented under the target model.

\subsection{Eliminating Roll-up Incompleteness}

In line with the general analysis concerning optional properties for conceptual design in [1] we argue that multiplicities of 0 should be avoided in understandable 


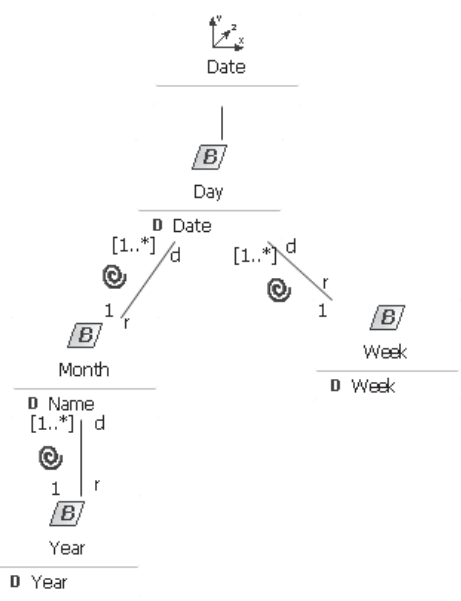

(a) Normalized Date dimension.

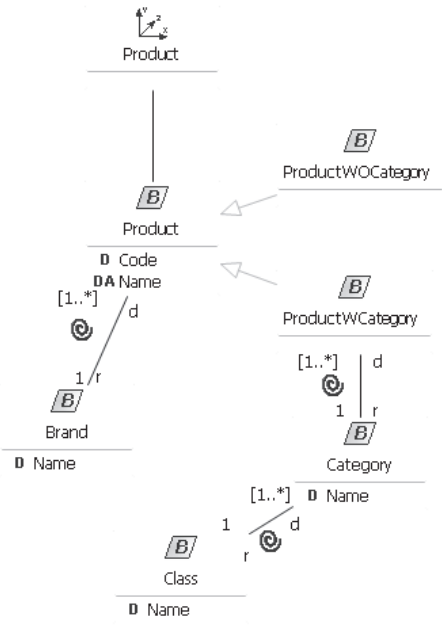

(b) Normalized Product dimension.

Fig. 4. Examples of normalized hierarchies.

conceptual models whenever possible. Instead, we advocate to apply generalization for conceptual MD modeling as well. To eliminate roll-up incompleteness, the transformation is based on one QVT relation that replaces all occurrences of roll-up incomplete Rolls-UpTo associations in the source model with generalization constructs. In this case, the optional Base class (which has multiplicity 0 at the role $r$ ) should be associated with a suitable sub-class in a generalization between Base classes: one to reflect instances with the optional property and the other one to reflect instances without that property.

The corresponding QVT relation (rollUpIncompleteBases) is shown in Fig. 3(b). It checks roll-up incompleteness in the Rolls-UpTo association between Base classes in the source model. Specifically, if a 0 multiplicity is detected in the role $r$ of a Rolls-UpTo association $s$ between two Base classes $b_{1}$ (e.g., Product in Fig. 1) and $b_{2}$ (e.g., Category in Fig. 1), then the relation enforces the creation of new elements in the target model as follows: Two new Base classes $b_{1} p$ and $b_{2} p$ that correspond to the source Base classes $b_{1}$ and $b_{2}$, respectively. In addition, two new Base sub-classes of $b_{1} p$, namely $b_{3}$ and $b_{4}$, are created via new generalization relationships $g_{1}$ and $g_{2}$. Here, $b_{3}$ reflects the instances of its super-class $b_{1} p$ that are associated with some instance of the optional Base class $b_{2} p$, and $b_{4}$ reflects the remaining instances of $b_{1} p$. Furthermore, the roll-up incomplete association $s$ between $b_{1}$ and $b_{2}$ is replaced with a roll-up complete association $r$ between $b_{3}$ and $b_{2} p$.

After the execution of the rollUpIncompleteBases relation, an excerpt of the target model related to the Product dimension is shown in Fig. 4(b). Here, two new Base classes, ProductWCategory and ProductWOCategory, are created to reflect those products that belong to a category or not, respectively. Again, those 
parts of the source model that do not present roll-up incompleteness problems are copied directly to the target model.

With respect to the soundness of the rollUpIncompleteBases relation, we focus on the roll-up incomplete association of the source model, say association $s$ between $b_{1}$ in the role $d$ and $b_{2}$ in the role $r$, which is represented differently in the target model. First, $b_{1}$ and $b_{2}$ are still present in the target model (as $b_{1} p$ and $\left.b_{2} p\right)$. Moreover, if an instance of $b_{1}$ is not related to any instance of $b_{2}$ (which exemplifies the incompleteness of the association) then this instance is simply stored as an instance of the same class under the target model (i.e. $b_{1} p$ ) and as an instance of its subclass $b_{4}$. If, however, an instance $i_{1}$ of $b_{1}$ is related to some instance $i_{2}$ of $b_{2}$ in the source model, then in the target model $i_{1}$ will be an instance of $b_{1} p$ and simultaneously an instance of $b_{1} p$ 's sub-class $b_{3}$. Now, this sub-class $b_{3}$ has a roll-up complete association with $b_{2} p$, which allows to retrieve the associated instance $i_{2}$ in the target model. Hence, the information that can be represented under the source model can also be represented under the target model.

\subsection{Eliminating Drill-down Incompleteness}

The transformation that eliminates drill-down incompleteness is based on one QVT relation that replaces all occurrences of drill-down incomplete Rolls-UpTo associations with normalized elements Due to space constraints this transformation is not shown, but the rationale of this QVT relation is removing summarizability problems by using generalization constructs. In this way, the optional Base class (which has multiplicity 0 at the role $d$, e.g., Region in Fig. 1) should be associated with a sub-class in a generalization between Base classes.

\section{Conclusions and Future Work}

Data warehouse designers have to make a great effort in defining dimension hierarchies that accurately reflect real-world situations in a MD model, whilst summarizability problems are avoided. In this paper, we have described a normalization approach for ensuring that the implemented MD model will be queried without the summarizability problems derived from the dimension hierarchies. Following our approach, designers can define dimension hierarchies that violate summarizability conditions in a conceptual model by using our UML profile. This conceptual model reflects real-world situations in an understandable way. Later, several transformations can be applied to automatically obtain a normalized MD model whose dimension hierarchies do not allow situations that attempt against summarizability, thus avoiding erroneous analysis of data.

Finally, we remark that the multidimensional normal forms defined in [2], which formalize quality aspects of MD models, deal with schemata with an expressiveness that is similar to the one of our normalized models. However, so far there is no work that considers the definition of such normal forms for semantically rich conceptual models. As an avenue for future work it appears 
attractive to define normal forms at the level of our normalized model: The generalization constructs included in this level enable a simplified treatment of optional levels (in [2], generalization is "simulated" by a careful application of context dependencies).

Our planned future work consists of evaluating and giving mechanisms to deal with the notion of "type compatibility" for summarizability of [4], which checks that the statistical function associated with the measure is summarizable according to the type of the measure and the type of the related dimensions.

Acknowledgments. This work has been partially supported by the following projects: SERENIDAD (PEII-11-0327-7035) from Junta de Comunidades de Castilla-La Mancha (Spain) and by the MESOLAP (TIN2010-14860) project from the Spanish Ministry of Education and Science.

\section{References}

1. Bodart, F., Patel, A., Sim, M., Weber, R.: Should optional properties be used in conceptual modelling? a theory and three empirical tests. Info. Sys. Research 12(4), 384-405 (2001)

2. Lechtenbörger, J., Vossen, G.: Multidimensional normal forms for data warehouse design. Inf. Syst. 28(5), 415-434 (2003)

3. Lehner, W., Albrecht, J., Wedekind, H.: Normal forms for multidimensional databases. In: Rafanelli, M., Jarke, M. (eds.) SSDBM. pp. 63-72. IEEE Computer Society (1998)

4. Lenz, H.J., Shoshani, A.: Summarizability in OLAP and statistical data bases. In: Ioannidis, Y.E., Hansen, D.M. (eds.) SSDBM. pp. 132-143. IEEE Computer Society (1997)

5. Luján-Mora, S., Trujillo, J., Song, I.Y.: A UML profile for multidimensional modeling in data warehouses. Data Knowl. Eng. 59(3), 725-769 (2006)

6. Malinowski, E., Zimányi, E.: Hierarchies in a multidimensional model: From conceptual modeling to logical representation. Data Knowl. Eng. 59(2), 348-377 (2006)

7. Malinowski, E., Zimányi, E.: Advanced data warehouse design: From conventional to spatial and temporal applications. Springer-Verlag (2008)

8. Mazón, J.N., Lechtenbörger, J., Trujillo, J.: Solving summarizability problems in fact-dimension relationships for multidimensional models. In: Song, I.Y., Abelló, A. (eds.) DOLAP. pp. 57-64. ACM (2008)

9. Mazón, J.N., Lechtenbörger, J., Trujillo, J.: A survey on summarizability issues in multidimensional modeling. Data Knowl. Eng. 68(12), 1452-1469 (2009)

10. Pedersen, T.B., Jensen, C.S., Dyreson, C.E.: Extending practical pre-aggregation in on-line analytical processing. In: VLDB. pp. 663-674 (1999)

11. Pedersen, T.B., Jensen, C.S., Dyreson, C.E.: A foundation for capturing and querying complex multidimensional data. Inf. Syst. 26(5), 383-423 (2001)

12. Rafanelli, M., Shoshani, A.: STORM: A statistical object representation model. In: Michalewicz, Z. (ed.) SSDBM. Lecture Notes in Computer Science, vol. 420, pp. 14-29. Springer (1990)

13. Rizzi, S., Abelló, A., Lechtenbörger, J., Trujillo, J.: Research in data warehouse modeling and design: dead or alive? In: Song, I.Y., Vassiliadis, P. (eds.) DOLAP. pp. 3-10. ACM (2006) 